\title{
Solvent-dependent stability of highly defective UiO-66 to acids and bases Daniele Cartagenova, ${ }^{a}$ Fabio A. Peixoto Esteves, ${ }^{a}$ Nathan T. Fischer, ${ }^{a}$ Jeroen A. van Bokhoven, ${ }^{a, b}$ Marco Ranocchiari*a
}

a Laboratory for Catalysis and Sustainable Chemistry, Paul Scherrer Institut, Forschungsstrasse 111, 5232 Villigen PSI, Switzerland.

${ }^{b}$ Department of Chemistry and Applied Biosciences, ETH Zürich, Vladimir Prelog Weg 1-5, 8093 Zurich, Switzerland.

*E-mail: marco.ranocchiari@psi.ch

\begin{abstract}
UiO-66 is one of the most chemically stable Metal-organic frameworks (MOFs) available. However, little is known about its stability in organic solvents. In this study, we synthesized a highly defective UiO-66 (HDUiO-66) and explored how its textural properties change when exposed to weak and strong acids, both organic and inorganic in nature, and dissolved in different solvents, water, dichloromethane (DCM), and tetrahydrofuran (THF). Exposing defective UiO-66 to weak acids and bases, such as acetic acid and triethylamine, maintains its crystalline structure and porosity, irrespective of the solvent. Sulphuric acid decomposes HD-UiO-66 in organic solvents but not in water, trifluoroacetic acid decomposes the framework only in DCM. Tetramethylguanidine decomposes HD-UiO-66 in organic solvents but mantains some of the MOFs porosity and crystalline structure in water, whereas potassium carbonate damages the MOF to a greater extent in water than in organic solvents. Our results show that the acid/base properties of the solvent modulate the strength of acids and bases and its polarity determines the extent of their solvation, thus playing a crucial role in altering the MOF's textural properties. This systematic investigation highlights the central role played by the solvent in tuning the stability of MOFs, which is relevant for liquidphase applications in acidic and basic environments, such as catalysis and adsorption.
\end{abstract}




\section{Introduction}

Metal-organic frameworks (MOFs) are porous coordination polymers constituted by inorganic nodes and organic linkers. ${ }^{1}$ Their unique features have drawn attention for applications in many different fields, including catalysis, gas separation and storage, and drug-delivery. ${ }^{2}$ A MOF of particular interest for the scientific community is UiO-66, first reported by Cavka et al. in $2008,{ }^{3}$ consisting of $\left[\mathrm{Zr}_{6}(\mathrm{OH})_{4} \mathrm{O}_{4}\right]^{12+}$ nodes and terephthalate linkers. Its thermal, ${ }^{4}$ chemical $^{5}$ and mechanical ${ }^{6}$ stability is attributed to the high connectivity and nuclearity of the inorganic node and promoted the development of a large family of " $\mathrm{Zr}_{6}$ MOFs", including $\mathrm{NU}-1000^{7}$ and $\mathrm{MOF}-808,{ }^{8}$ with tetradentate and tridentate carboxylate linkers, respectively. Isoreticular MOFs were synthesized by using linkers with various functional groups ${ }^{4}$ and different size, ${ }^{3}$ post-synthetic modification (PSM), ${ }^{9,10}$ and post-synthetic exchange (PSE) of the linker ${ }^{11}$ and of the nodes. ${ }^{12}$

The simulated BET surface area of a defect-free UiO-66 structure is $1100 \mathrm{~m}^{2} / \mathrm{g} .{ }^{13}$ The addition of acids as modulators during the synthesis of the framework promotes the formation of "missing linker" and/or "missing cluster" defects. ${ }^{14-17}$ Defects result in different properties of the framework, such as increased chemical reactivity in $\mathrm{PSM},{ }^{18}$ increased Lewis acidity, ${ }^{19}$ increased surface area ${ }^{20}$ and improved gas sorption properties, ${ }^{21}$ but may cause a reduction in the thermal stability of the compound..$^{22}$

MOFs can decompose upon interaction with water via hydrolysis of the metal-linker bond. ${ }^{23}$ This phenomenon is extremely important for gas phase applications, such as carbon dioxide and water capture, and the stability of MOFs towards water vapour has been studied extensively. ${ }^{24}$ Similar mechanisms of decomposition may take place in liquid phase applications, such as catalysis. UiO-66 is stable upon treatment with aqueous solutions of different $\mathrm{pH}$ and with common solvents, retaining its crystalline structure. ${ }^{4,5,25}$ This may not be due necessarily to the framework's inertness, but rather to a dynamic process of ligand exchange. ${ }^{11}$ More recently, Leus et al. proved that a significant loss of the 
porosity of MOFs can take place without affecting their diffraction patterns, ${ }^{26}$ highlighting how nitrogen physisorption and diffraction provide complementary insight into the stability of MOFs upon chemical treatment. Bůžek et al. reported a detailed investigation of the stability of UiO-66 to aqueous buffer solutions. ${ }^{27}$ The reactivity of UiO-66 with acidic and basic solutions in organic solvents is extremely important for its applications. Its stability was, for the most part, proved indirectly: UiO-66- $\mathrm{NH}_{2}$ underwent PSM treatments with anhydrides both in solution of dichloromethane or chloroform or in gas phase, preserving crystallinity and porosity $;^{9,10,28}$ and a UiO-67 derivative functionalized with palladium retained its structure after being used in Suzuki-Miyaura coupling. ${ }^{29}$ The role that defects in UiO-66 play in the chemical stability towards acids, bases and solvents remains largely unexplored.

In this work, we synthesized a highly defective UiO-66 (HD-UiO-66) and studied its reactivity towards acids and bases in different solvents. By studying the textural properties of the samples with nitrogen physisorption and powder X-ray diffraction, we demonstrate that the solvent has a dramatic influence on modulating the interaction of the framework with such chemicals.

\section{Results}

We synthesized HD-UiO-66 with acetic acid as a modulator, giving a crystalline material with PXRD pattern showing a UiO-66 topology (Figure S1, ESI) and with a BET surface area larger than $2000 \mathrm{~m}^{2} / \mathrm{g}$ (Figure S2, ESI), among the highest values reported for this material. ${ }^{30}$ Thermal Gravimetric Analysis (TGA) shows a linker-to-node ratio of around 4.1, indicative of a high defectivity of the sample. The TGA curve (Figure S3, ESI) shows the decomposition of the framework between 480 and $500^{\circ} \mathrm{C}$, comparable to that reported elsewhere. ${ }^{14}$ We explored how the textural properties of HD-UiO-66 would change, depending (a) on the polarity of the solvent from protic (water), to aprotic and polar (THF) and apolar (DCM) and (b) on the nature and strength of the acid and the base from inorganic $\left(\mathrm{H}_{2} \mathrm{SO}_{4}\right.$ and $\mathrm{K}_{2} \mathrm{CO}_{3}$ ), strong organic (TFA and $\mathrm{TMG})$ and weak organic $\left(\mathrm{AcOH}\right.$ and $\left.\mathrm{Et}_{3} \mathrm{~N}\right)$. 


\section{Treatment of HD-UiO-66 in aqueous solutions}

The PXRD of the material formed after treatment of HD-UiO-66 with pure water showed reflections belonging to the UiO-66 framework, broader and of lower intensity than in the pristine material (Figure 1, left, red and black curves). The BET surface area of the material reduced to $1590 \mathrm{~m}^{2} / \mathrm{g}$ (Table S1, entry 2, ESI), and the nitrogen adsorption isotherm showed a lower uptake than the pristine material at $\mathrm{p} / \mathrm{p}_{0}>0.1$ (Figure 1, right, red and black squares).

Treating the MOF with $0.1 \mathrm{M} \mathrm{H}_{2} \mathrm{SO}_{4}$ gave an almost identical PXRD pattern as that of the parent material (Figure 1, left, blue curve). The nitrogen physisorption isotherm featured a steeper increase at $\mathrm{p} / \mathrm{p}_{0}>0.1$ than the pristine material and resulted in a BET surface area of $1630 \mathrm{~m}^{2} / \mathrm{g}$ (Figure 1, right, blue squares). The material after exposure to a 0.1 M TFA solution showed an increased baseline in the PXRD pattern (Figure 1, left, green curve); the nitrogen adsorption isotherm maintained a similar shape to the starting material but with reduced uptake capacity and a BET surface area of $1700 \mathrm{~m}^{2} / \mathrm{g}$ (Figure 1, right, green squares). Exposure to 0.1 M AcOH resulted only in slight PXRD peak broadening (Figure 1, left, light blue curve), and the nitrogen adsorption capacity was reduced by a small extent; the BET surface area was $1820 \mathrm{~m}^{2} / \mathrm{g}$ (Figure 1, left, light blue squares).

Upon exposure to $0.1 \mathrm{M}$ aqueous $\mathrm{K}_{2} \mathrm{CO}_{3}$ the sample lost its diffraction pattern (Figure 2, left, blue curve), and the nitrogen uptake capacity was significantly reduced, with a BET surface area of $540 \mathrm{~m}^{2} / \mathrm{g}$ (Figure 2, right, blue squares). The diffraction pattern of the sample treated with a 0.1 M TMG solution showed only a few broad reflections, corresponding to the most intense signals (Figure 2, left, green curve), and the BET surface area was reduced to $980 \mathrm{~m}^{2} / \mathrm{g}$ (Figure 2, right, green squares). The treatment with $0.1 \mathrm{M}$ aqueous Et ${ }_{3} \mathrm{~N}$ caused only a slight reduction in the intensity of the PXRD signals and a minor increase in the baseline (Figure 2, left, light blue), but the nitrogen physisorption isotherm was similar to that of the previous sample, with a BET surface area of $1020 \mathrm{~m}^{2} / \mathrm{g}$ (Figure 2, right, light blue squares). 


\section{Treatment of HD-UiO-66 in tetrahydrofuran solutions}

The treatment of HD-UiO-66 with pure THF resulted in a slight broadening of the PXRD peaks (Figure 3, left, red curve) and a decrease in the nitrogen uptake, with a BET surface area of $1620 \mathrm{~m}^{2} / \mathrm{g}$ (Figure 3, right, red squares).

Treating HD-UiO-66 with a $0.1 \mathrm{M}$ solution of $\mathrm{H}_{2} \mathrm{SO}_{4}$ in THF caused the loss of the diffraction pattern (Figure 3, left, blue curve) and of the nitrogen uptake capacity of the material, resulting in a BET surface area of $50 \mathrm{~m}^{2} / \mathrm{g}$ (Figure 3, right, blue squares). The material obtained after exposure to a TFA solution had a diffraction pattern comparable to that of the pristine material (Figure 3, left, green curve) and a similar nitrogen physisorption isotherm, with a BET surface area of $1510 \mathrm{~m}^{2} / \mathrm{g}$ (Figure 3, right, green squares). HD-UiO-66 treated with 0.1 M AcOH showed a minimally affected PXRD pattern (Figure 3, left, light blue curve) and nitrogen physisorption isotherm, with a BET surface area of $1700 \mathrm{~m}^{2} / \mathrm{g}$ (Figure 3, right, light blue squares).

The PXRD pattern of HD-UiO-66 after treatment with $0.1 \mathrm{M} \mathrm{K}_{2} \mathrm{CO}_{3}$ in THF was similar to that of the starting material but with two additional reflections, attributed to residues of the base (Figure 4, left, blue curve). The BET surface area was reduced to $1060 \mathrm{~m}^{2} / \mathrm{g}$ (Figure 4, right, blue squares), a value that is underestimated because of the presence of a non-porous solid in the mixture. The material obtained after exposure of the MOF to a TMG solution showed only a broad reflection in the diffractogram (Figure 4, left, green curve) and a low nitrogen physisorption isotherm, with a BET surface area of $470 \mathrm{~m}^{2} / \mathrm{g}$ (Figure 4, right, green squares). Treatment with $0.1 \mathrm{M} \mathrm{Et}_{3} \mathrm{~N}$ did not affect the PXRD pattern (Figure 4, left, light blue curve) and caused only a slight reduction in nitrogen uptake, with a decrease in BET surface area to $1760 \mathrm{~m}^{2} / \mathrm{g}$ (Figure 4, right, light blue squares). 


\section{Treatment of HD-UiO-66 in dichloromethane solutions}

Soaking HD-UiO-66 in pure DCM had a minimal effect on its diffraction pattern (Figure 5, left, red curve) and nitrogen physisorption isotherm, with a reduction of the BET surface area to $1850 \mathrm{~m}^{2} / \mathrm{g}$ (Figure 5 , right, red squares).

The material that formed after treatment of HD-UiO-66 with $0.1 \mathrm{M} \mathrm{H}_{2} \mathrm{SO}_{4}$ in this solvent had different PXRD pattern than the starting material (Figure 5, left, blue curve) and showed no nitrogen uptake, with a BET surface area of $70 \mathrm{~m}^{2} / \mathrm{g}$ (Figure 5 , right, blue squares). Exposure to a TFA solution resulted in a diffraction pattern with weak reflections belonging to the starting material and a broad signal between $5^{\circ}$ and $9^{\circ}$ (Figure 5, left, green curve) and the BET surface area decreased to $680 \mathrm{~m}^{2} / \mathrm{g}$ (Figure 5 , right, green squares). After treatment with $0.1 \mathrm{M} \mathrm{AcOH}$, the material retained its PXRD pattern (Figure 5, left, light blue curve) and nitrogen physisorption isotherm, with a BET surface area of $1950 \mathrm{~m}^{2} / \mathrm{g}$ (Figure 5, right, light blue squares).

The diffraction pattern of the material obtained after treating $\mathrm{HD}$-UiO-66 with $\mathrm{K}_{2} \mathrm{CO}_{3}$ in $\mathrm{DCM}$ contains weak and broad reflections of the starting material as well as those of the base residues (Figure 6, left, blue curve); its BET surface area was reduced to $680 \mathrm{~m}^{2} / \mathrm{g}$ (Figure 6 , right, blue squares), an underestimated value. Treatment with a 0.1 M TMG solution resulted in the loss of the framework reflections from the PXRD pattern, which contains only a broad peak (Figure 6, left, green curve), and in a lower nitrogen physisorption isotherm with a BET surface area of $640 \mathrm{~m}^{2} / \mathrm{g}$ (Figure 6 , right, green squares). Exposing the MOF to a $\mathrm{Et}_{3} \mathrm{~N}$ solution did not change the PXRD pattern (Figure 6, left, light blue curve) and reduced the BET surface area to $1710 \mathrm{~m}^{2} / \mathrm{g}$ (Figure 6, right, light blue squares).

\section{Discussion}

Figure 7 schematically summarizes our observations. The textural properties of the MOF are impacted considerably, both by the solvent and the presence of an acid or a base. HD-UiO-66 shows similar stability 
in water as the corresponding defect-free material, being more resistant to acidic than to basic solutions. ${ }^{4,}$

${ }^{5}$ By comparing the effect of solvents alone, we found that polar solvents have a stronger effect on reduction of crystallite size, mesoporosity (Figure 1, 3 and 5, red samples) and BET surface area (Table S1, entries 2, 9 and 16, ESI). As Kim et al. demonstrated, suspension in solvents promotes the dissolution and recrystallization of this material, with a higher rate found for polar solvents. ${ }^{11}$

While HD-UiO-66 is stable after treatment with weak acids and bases in all solvents, its textural properties are strongly affected by the solvent in the treatment with strong acids and bases. In comparing the effect of strong acid solutions in water and in DCM, it is clear that $\mathrm{H}_{2} \mathrm{SO}_{4}$ and TFA have a minimal effect on the integrity of HD-UiO-66 in aqueous solution, losing around 20\% of its surface area (Table S1, entries 3 and 4, ESI), while in DCM there is decomposition of the MOF (Figure 5, blue and green curves). $\mathrm{Both}_{2} \mathrm{SO}_{4}$ and TFA dissociate to $\mathrm{H}_{3} \mathrm{O}^{+}$and the corresponding conjugated base in water. The acid $\mathrm{H}_{3} \mathrm{O}^{+}$is the one that likely reacts with the framework. The situation is different in the apolar solvent DCM, which does not have basic sites. In that case the strongest proton acceptor in the reaction mixture is the framework: protonation of terephthalate linkers by $\mathrm{H}_{2} \mathrm{SO}_{4}$ and TFA is a plausible explanation for the decomposition of the MOF in DCM. ${ }^{24}$ In THF, which is still polar but not protic, the results were intermediate: $\mathrm{a}_{2} \mathrm{SO}_{4}$ solution causes the decomposition of the framework, while a TFA solution has only minor effects on its textural properties (Figure 3, blue and green curves).

The situation with the textural properties of HD-UiO-66 treated with basic water solutions is more complex. TMG has a $\mathrm{pK}_{\mathrm{a}}$ value of 13 , while $\mathrm{K}_{2} \mathrm{CO}_{3}$ and $\mathrm{Et}_{3} \mathrm{~N}$ have a similar $\mathrm{pK}_{\mathrm{a}}$, around 10.5 in water, showing that stability does not depend exclusively on the strength of the base. Even though TMG is a much stronger base than $\mathrm{K}_{2} \mathrm{CO}_{3}$, it damages the framework to a lesser extent and reduces the surface area as much as a solution of $\mathrm{Et}_{3} \mathrm{~N}$. This shows that the nature and size of the base play important roles in the reactivity of $\mathrm{HD}-\mathrm{UiO}-66$ in water and that the concentration of $\mathrm{OH}^{-}$is not the only determining factor in MOF decomposition. Whereas water solutions of the organic bases have a similar effect on the 
framework, in other solvents the effects differ significantly. Solutions of TMG in organic solvents destroy the framework; solutions of $\mathrm{Et}_{3} \mathrm{~N}$ leave it unaffected. The different basic strengths may not be the only explanation; other properties of the reaction mixture, e.g. the ligand properties of the bases and how they are affected by solvation, must be considered. Water, with its hydrogen-bond donor capacity, can efficiently solvate the bases and modulate their interaction with zirconium ions. In solution of THF or DCM, the bases are "naked" and can interact directly with the framework's nodes.

In conclusion, the stability of HD-UiO-66 is solvent-dependent especially upon treatment with strong acids and strong bases. The role of the solvent is to solvate the acid or base and their conjugated species and act as a mediator of the acid and base strength. Whereas in water the acids and bases produce $\mathrm{H}^{+}$and $\mathrm{OH}^{-}$ this is not the case in organic solvents, which leave the acids and bases more "naked", enabling different reactivity with the framework. The treatment of HD-UiO-66 with mild acids and bases minimally changes its textural properties in all solvents. These results will be useful in the MOF community, since the conditions studied are relevant for catalysis and other liquid-phase applications. 

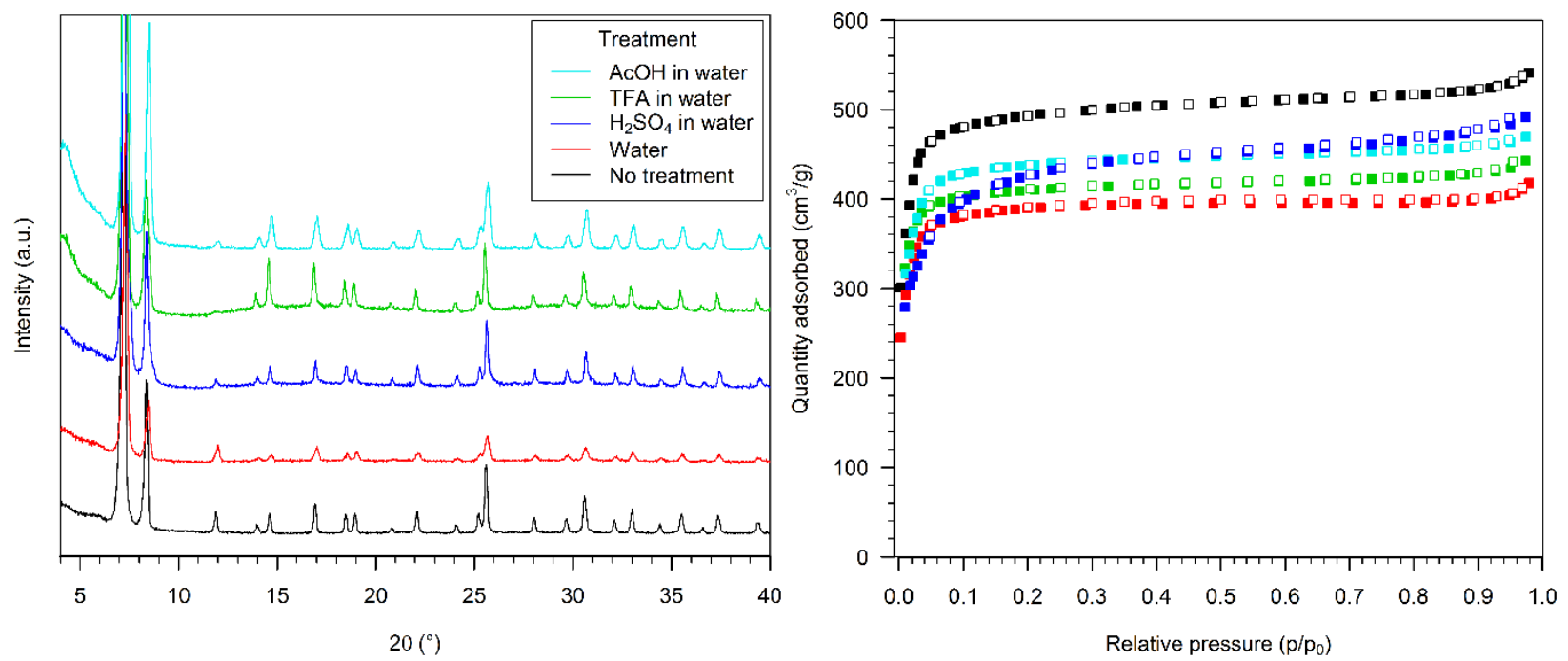

Figure 1: (left) PXRD patterns of as synthesized HD-UiO-66 (black) and of HD-UiO-66 after treatment with water (red) and with acidic aqueous solutions of $\mathrm{H}_{2} \mathrm{SO}_{4}$ (blue), TFA (green) and AcOH (light blue). (right) Corresponding nitrogen physisorption isotherms, adsorption represented by filled squares, desorption by open squares. 

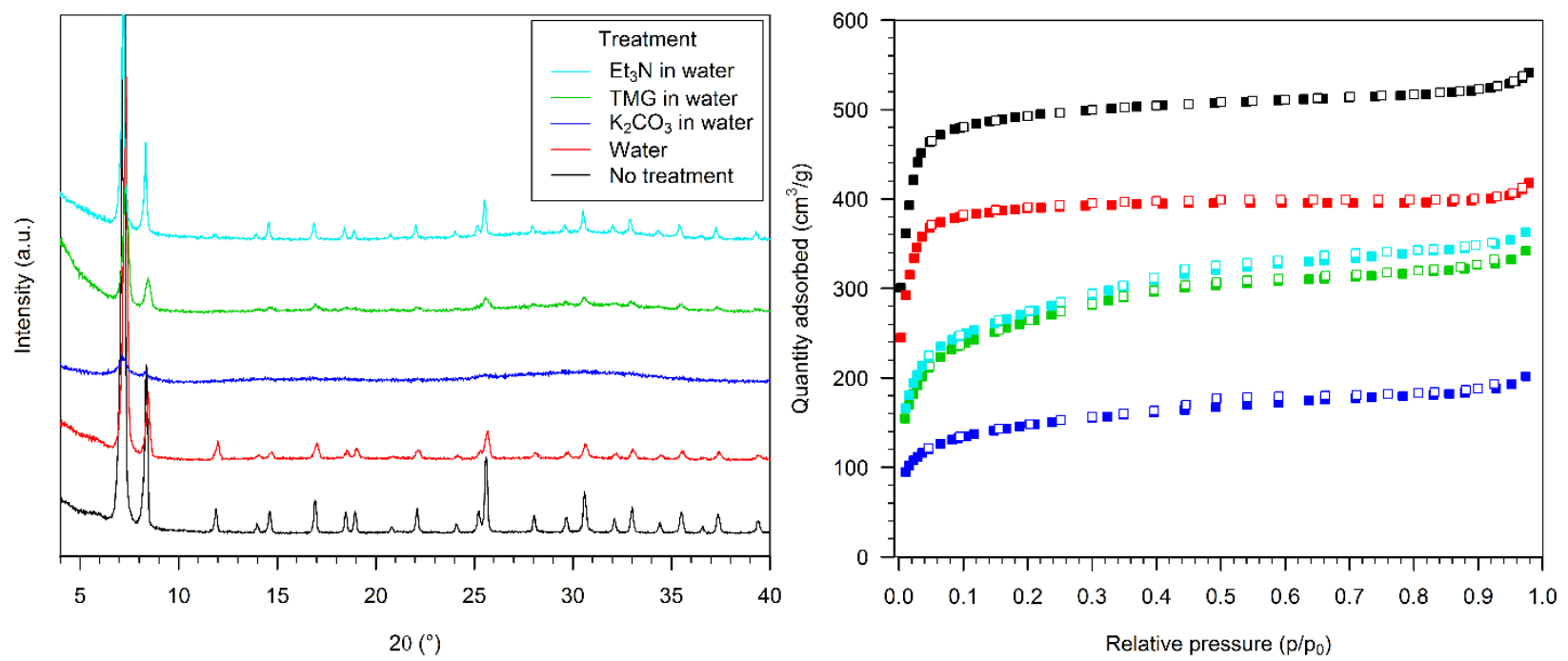

Figure 2: (left) PXRD patterns of as synthesized HD-UiO-66 (black) and of HD-UiO-66 after treatment with water (red) and with basic aqueous solutions of $\mathrm{K}_{2} \mathrm{CO}_{3}$ (blue), $\mathrm{TMG}$ (green) and $\mathrm{Et}_{3} \mathrm{~N}$ (light blue). (right) Corresponding nitrogen physisorption isotherms, adsorption represented by filled squares, desorption by open squares. 

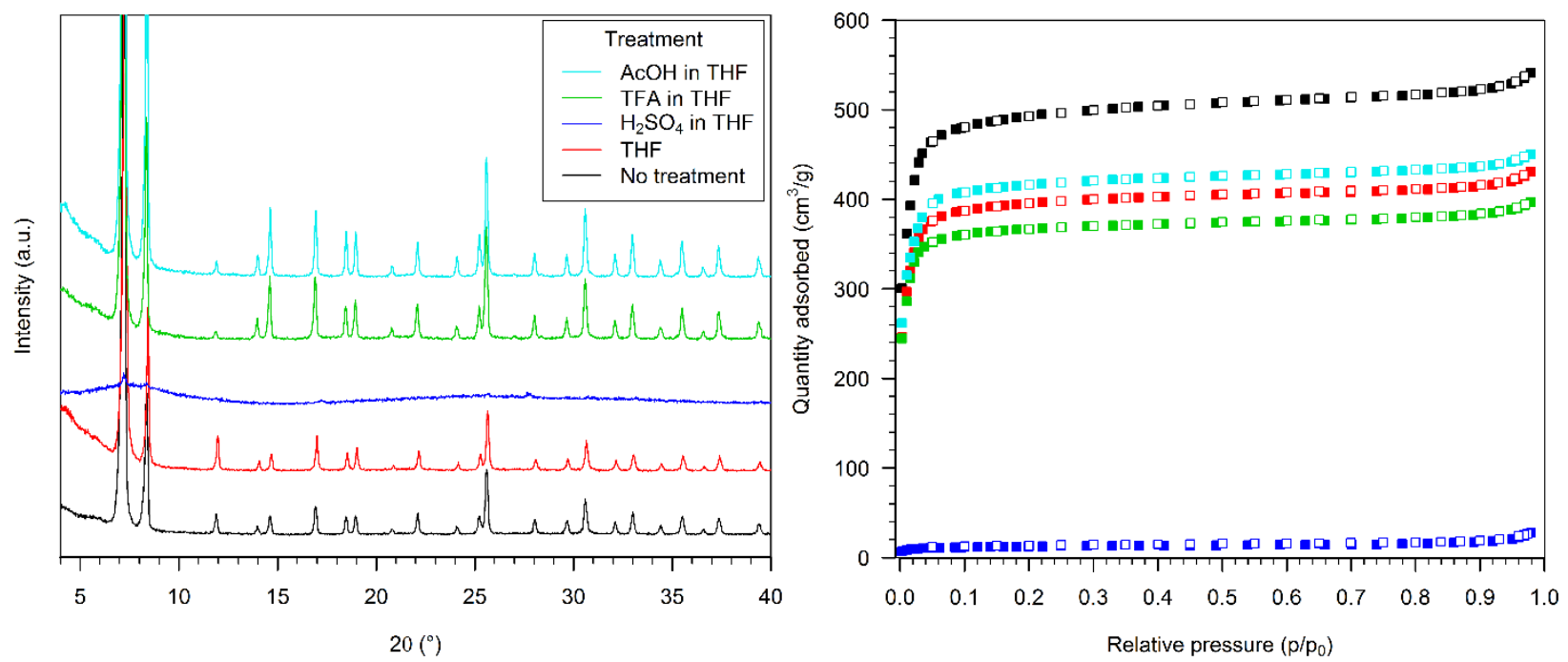

Figure 3: (left) PXRD patterns of as synthesized HD-UiO-66 (black) and of HD-UiO-66 after treatment with THF (red) and with acidic solutions of $\mathrm{H}_{2} \mathrm{SO}_{4}$ (blue), TFA (green) and $\mathrm{AcOH}$ (light blue) in THF. (right) Corresponding nitrogen physisorption isotherms, adsorption represented by filled squares, desorption by open squares. 

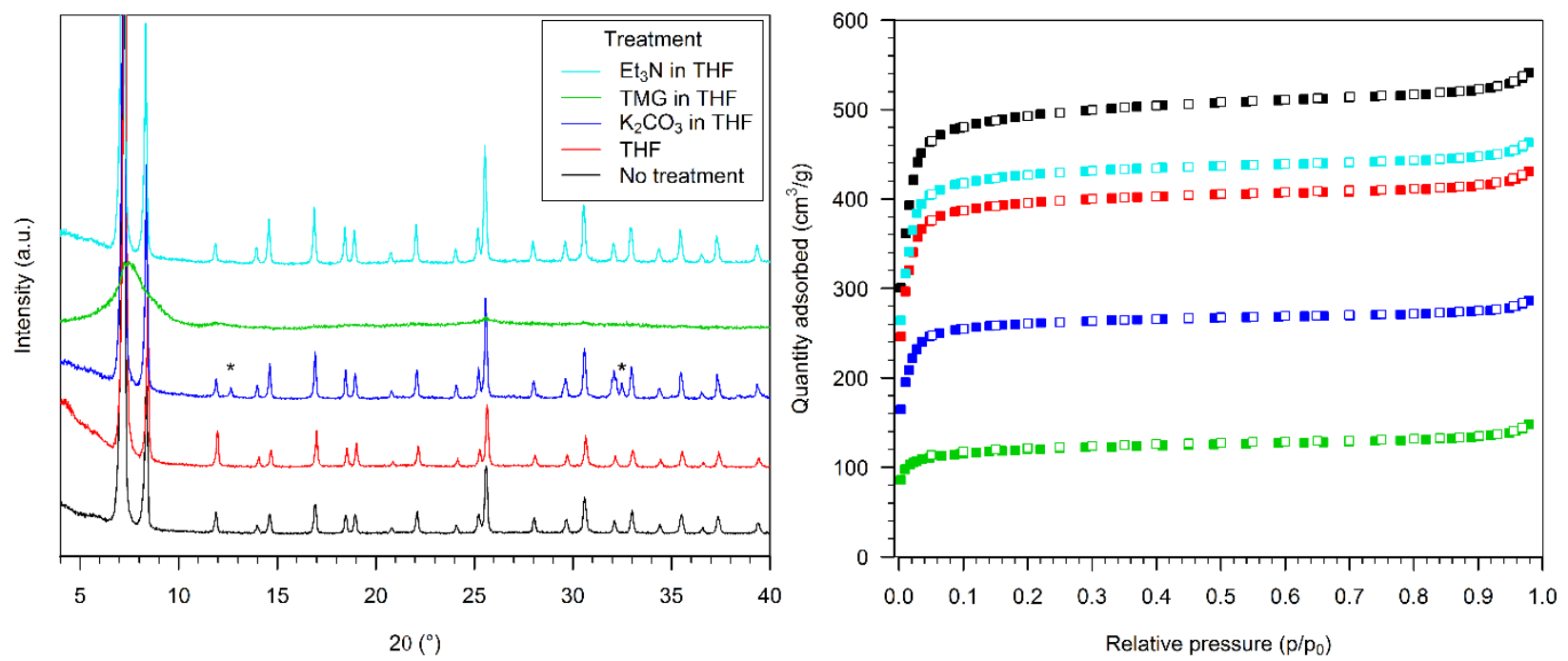

Figure 4: (left) PXRD patterns of as synthesized HD-UiO-66 (black) and of HD-UiO-66 after treatment with THF (red) and with basic solutions of $\mathrm{K}_{2} \mathrm{CO}_{3}$ (blue), $\mathrm{TMG}$ (green) and $\mathrm{Et}_{3} \mathrm{~N}$ (light blue) in THF. Residues of $\mathrm{K}_{2} \mathrm{CO}_{3}$ are indicated by a star. (right) Corresponding nitrogen physisorption isotherms, adsorption represented by filled squares, desorption by open squares. 

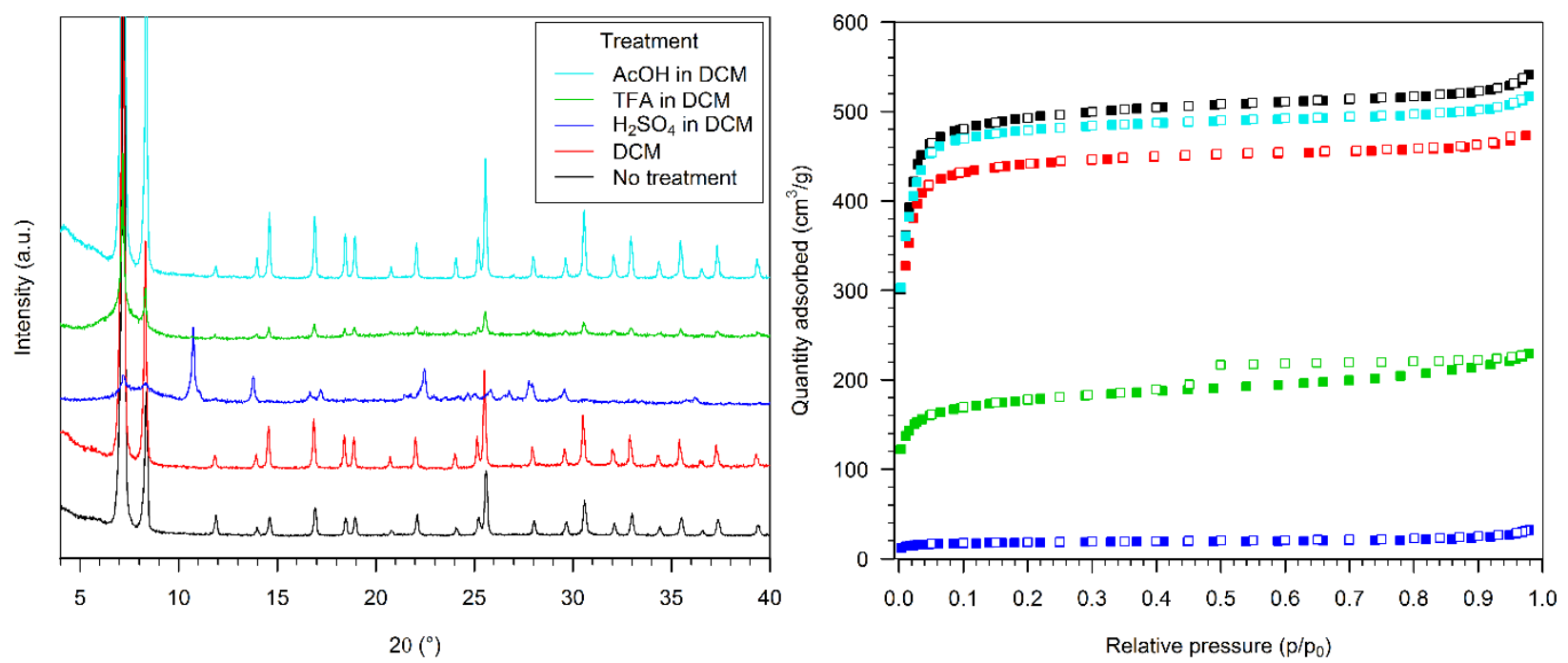

Figure 5: (left) PXRD patterns of as synthesized HD-UiO-66 (black) and of HD-UiO-66 after treatment with DCM (red) and with acidic solutions of $\mathrm{H}_{2} \mathrm{SO}_{4}$ (blue), TFA (green) and $\mathrm{AcOH}$ (light blue) in DCM. (right) Corresponding nitrogen physisorption isotherms, adsorption represented by filled squares, desorption by open squares. 

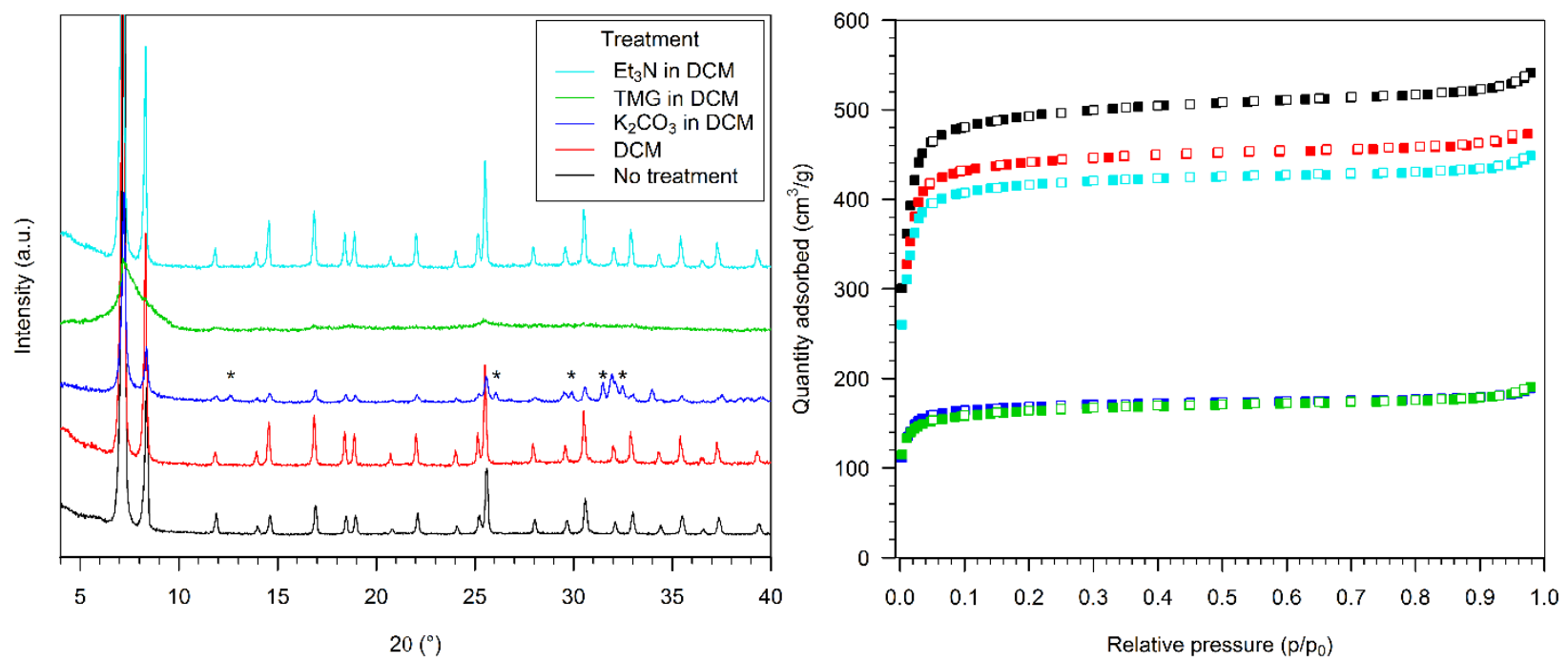

Figure 6: (left) PXRD patterns of as synthesized HD-UiO-66 (black) and of HD-UiO-66 after treatment with DCM (red) and with basic solutions of $\mathrm{K}_{2} \mathrm{CO}_{3}$ (blue), TMG (green) and $\mathrm{Et}_{3} \mathrm{~N}$ (light blue) in DCM. Residues of $\mathrm{K}_{2} \mathrm{CO}_{3}$ are indicated by a star. (right) Corresponding nitrogen physisorption isotherms, adsorption represented by filled squares, desorption by open squares. 


\begin{tabular}{|c|c|c|c|c|c|c|c|c|c|c|c|c|c|c|}
\hline \multirow{2}{*}{$\begin{array}{l}\text { Acid / } \\
\text { base } \\
\text { Solvent }\end{array}$} & \multicolumn{2}{|l|}{-} & \multicolumn{2}{|c|}{$\mathrm{H}_{2} \mathrm{SO}_{4}$} & \multicolumn{2}{|c|}{ TFA } & \multicolumn{2}{|c|}{$\mathrm{AcOH}$} & \multicolumn{2}{|c|}{$\mathrm{K}_{2} \mathrm{CO}_{3}$} & \multicolumn{2}{|c|}{ TMG } & \multicolumn{2}{|c|}{$\mathrm{Et}_{3} \mathrm{~N}$} \\
\hline & $C$ & $P$ & $C$ & P & $C$ & $P$ & $C$ & $P$ & $C$ & $P$ & $C$ & $P$ & C & $P$ \\
\hline \multicolumn{15}{|l|}{ Water } \\
\hline \multicolumn{15}{|l|}{ THF } \\
\hline DCM & & & & & & & & & & & & & & \\
\hline
\end{tabular}

Figure 7: Summary on the retention of textural properties of HD-UiO-66, according to both characterization techniques. Colour gradient: green = complete retention and yellow = complete loss of textural properties. 


\section{References}

1. W. Lu, Z. Wei, Z.-Y. Gu, T.-F. Liu, J. Park, J. Park, J. Tian, M. Zhang, Q. Zhang, T. Gentle III, M. Bosch and H.-C. Zhou, Chem. Soc. Rev., 2014, 43, 5561-5593.

2. H. Furukawa, K. E. Cordova, M. O'Keeffe and O. M. Yaghi, Science, 2013, 341.

3. J. H. Cavka, S. Jakobsen, U. Olsbye, N. Guillou, C. Lamberti, S. Bordiga and K. P. Lillerud, J. Am. Chem. Soc., 2008, 130, 13850-13851.

4. M. Kandiah, M. H. Nilsen, S. Usseglio, S. Jakobsen, U. Olsbye, M. Tilset, C. Larabi, E. A. Quadrelli, F. Bonino and K. P. Lillerud, Chem. Mater., 2010, 22, 6632-6640.

5. J. B. DeCoste, G. W. Peterson, H. Jasuja, T. G. Glover, Y.-g. Huang and K. S. Walton, J. Mater. Chem. A, 2013, 1, 5642-5650.

6. H. Wu, T. Yildirim and W. Zhou, J. Phys. Chem. Lett., 2013, 4, 925-930.

7. J. E. Mondloch, W. Bury, D. Fairen-Jimenez, S. Kwon, E. J. DeMarco, M. H. Weston, A. A. Sarjeant, S. T. Nguyen, P. C. Stair, R. Q. Snurr, O. K. Farha and J. T. Hupp, J. Am. Chem. Soc., 2013, 135, 10294-10297.

8. H. Furukawa, F. Gándara, Y.-B. Zhang, J. Jiang, W. L. Queen, M. R. Hudson and O. M. Yaghi, J. Am. Chem. Soc., 2014, 136, 4369-4381.

9. M. Kandiah, S. Usseglio, S. Svelle, U. Olsbye, K. P. Lillerud and M. Tilset, J. Mater. Chem., 2010, 20, 9848-9851.

10. S. J. Garibay and S. M. Cohen, Chem. Commun., 2010, 46, 7700-7702.

11. M. Kim, J. F. Cahill, Y. Su, K. A. Prather and S. M. Cohen, Chem. Sci., 2012, 3, 126-130.

12. M. Kim, J. F. Cahill, H. Fei, K. A. Prather and S. M. Cohen, J. Am. Chem. Soc., 2012, 134, 1808218088.

13. M. J. Katz, Z. J. Brown, Y. J. Colón, P. W. Siu, K. A. Scheidt, R. Q. Snurr, J. T. Hupp and O. K. Farha, Chem. Commun., 2013, 49, 9449-9451.

14. G. C. Shearer, S. Chavan, S. Bordiga, S. Svelle, U. Olsbye and K. P. Lillerud, Chem. Mater., 2016, 28, 3749-3761.

15. M. Taddei, Coord. Chem. Rev., 2017, 343, 1-24.

16. M. Taddei, R. J. Wakeham, A. Koutsianos, E. Andreoli and A. R. Barron, Angew. Chem. Int. Ed. Engl., 2018, 57, 11706-11710.

17. L. Liu, Z. Chen, J. Wang, D. Zhang, Y. Zhu, S. Ling, K.-W. Huang, Y. Belmabkhout, K. Adil, Y. Zhang, B. Slater, M. Eddaoudi and Y. Han, Nat. Chem., 2019, 11, 622-628.

18. G. C. Shearer, J. G. Vitillo, S. Bordiga, S. Svelle, U. Olsbye and K. P. Lillerud, Chem. Mater., 2016, 28, 7190-7193.

19. F. Vermoortele, B. Bueken, G. Le Bars, B. Van de Voorde, M. Vandichel, K. Houthoofd, A. Vimont, M. Daturi, M. Waroquier, V. Van Speybroeck, C. Kirschhock and D. E. De Vos, J. Am. Chem. Soc., 2013, 135, 11465-11468.

20. H. Wu, Y. S. Chua, V. Krungleviciute, M. Tyagi, P. Chen, T. Yildirim and W. Zhou, J. Am. Chem. Soc., 2013, 135, 10525-10532.

21. A. W. Thornton, R. Babarao, A. Jain, F. Trousselet and F. X. Coudert, Dalton Trans., 2016, 45, $4352-4359$.

22. G. C. Shearer, S. Chavan, J. Ethiraj, J. G. Vitillo, S. Svelle, U. Olsbye, C. Lamberti, S. Bordiga and K. P. Lillerud, Chem. Mater., 2014, 26, 4068-4071.

23. J. J. Low, A. I. Benin, P. Jakubczak, J. F. Abrahamian, S. A. Faheem and R. R. Willis, J. Am. Chem. Soc., 2009, 131, 15834-15842.

24. S. Yuan, L. Feng, K. Wang, J. Pang, M. Bosch, C. Lollar, Y. Sun, J. Qin, X. Yang, P. Zhang, Q. Wang, L. Zou, Y. Zhang, L. Zhang, Y. Fang, J. Li and H.-C. Zhou, Adv. Mater., 2018, 30, 1704303. 
25. C. G. Piscopo, A. Polyzoidis, M. Schwarzer and S. Loebbecke, Microporous Mesoporous Mater., 2015, 208, 30-35.

26. K. Leus, T. Bogaerts, J. De Decker, H. Depauw, K. Hendrickx, H. Vrielinck, V. Van Speybroeck and P. Van Der Voort, Microporous Mesoporous Mater., 2016, 226, 110-116.

27. D. Bǔžek, S. Adamec, K. Lang and J. Demel, Inorg. Chem. Front., 2021, DOI: 10.1039/d0qi00973c.

28. M. Servalli, M. Ranocchiari and J. A. Van Bokhoven, Chem. Commun., 2012, 48, 1904-1906.

29. H. Fei and S. M. Cohen, Chem. Commun., 2014, 50, 4810-4812.

30. K. Wang, C. Li, Y. Liang, T. Han, H. Huang, Q. Yang, D. Liu and C. Zhong, Chem. Eng. J., 2016, 289, 486-493. 